\title{
Analysis of magnetopiezoelastic energy harvesters under random excitations: an equivalent linearization approach
}

\author{
S. F. Ali ${ }^{a}$, S. Adhikari ${ }^{b}$ and M. I. Friswell ${ }^{b}$ \\ ${ }^{a}$ Newton Fellow, College of Engineering, Swansea University, Swansea SA2 8PP, UK. \\ ${ }^{b}$ Proffesor, College of Engineering, Swansea University, Swansea SA2 8PP, UK.
}

\begin{abstract}
This paper develops an equivalent linear model for piezomagnetoelastic energy harvesters under broadband random ambient excitations. Piezomagnetoelastic harvesters are used for powering low power electronic sensor systems. Nonlinear behaviour arising due to the vibration in a magnetic field makes piezomagnetoelastic energy harvesters different from the more classical piezoelastic energy harvesters. First numerical simulation of the nonlinear model is presented and then an equivalent linearization based analytical approach is developed for the analysis of harvested power. A cosed-form approximate expression for the ensemble average of the harvested power is derived. The equivalent model is seen to capture the details of the nonlinear model and also provides more details to the behaviour of the harvester to random excitation. Our results show that it is possible to optimally design the system such that the mean harvested power is maximized for a given strength of the input broadband random ambient excitation.
\end{abstract}

Keywords: Nonlinear energy harvesting, Magnetopiezoelastic systems, statistical linearization

\section{INTRODUCTION}

The evolution of electronic devices since the beginning of micro and nano-electronics has brought about an exponential growth in computational power in ever shrinking systems and the associated increase in functionality brings a new revolution in systems targeting wearable healthcare, lifestyle and industrial monitoring applications.

As systems continue to shrink, less energy is required onboard. This allowed another energy paradigm of energy harvesting (also referred to as energy scavenging) from the environment. A decade of research in the field of energy harvesting has led to the efficient capturing of small amounts of energy from the environment and transforming them into electrical energy. Energy autonomous systems using energy harvesting are particularly attractive when long term remote deployment is needed or wherever a natural long term energy source is available (such as for example temperature or vibrations) for continuous replenishing of the energy consumed by the system. Such inexhaustible energy supply is a significant advantage over battery supply or mains power. Extended lifetime and autonomy are particularly advantageous in systems with limited accessibility, such as medical implants and infrastructure integrated micro-sensors, wireless sensor nodes used for structural health monitoring, embedded and implanted sensor nodes for medical applications, recharging the batteries of large systems, monitoring tire pressure in automobiles, powering unmanned vehicles, and running security systems in household conditions.

As described by Williams and Yates, ${ }^{1}$ the three basic vibration-to-electric energy conversion mechanisms are electromagnetic, ${ }^{1-4}$ electrostatic, ${ }^{5,6}$ and piezoelectric ${ }^{7-10}$ transductions. In the last decade, these transduction mechanisms have been investigated by numerous researchers for vibration-based energy harvesting and extensive discussions can be found in the existing review articles. ${ }^{5,9,11,12}$

Energy harvesting of ambient vibration is important for remote devices, for example in structural health monitoring. ${ }^{5,9,11-13}$ Completely wireless sensor systems are desirable and this can only be accomplished by using batteries and/or harvested energy. Harvesting is attractive because the energy generated can be used directly or used to recharge batteries or other storage devices, which enhances battery life. Most of the results using the piezoelectric effect as the transduction method have used cantilever beams and single frequency excitation, i.e.,

Further author information: (Send correspondence to Shaikh Faruque Ali)

E-mail: sk.faruque.ali@gmail.com

Active and Passive Smart Structures and Integrated Systems 2011, edited by Mehrdad N. Ghasemi-Nejhad,

Proc. of SPIE Vol. $7977,79770 N \cdot C 2011$ SPIE · CCC code: 0277-786X/11/\$18 · doi: 10.1117/12.880437

Proc. of SPIE Vol. 797779770 N-1 
resonance based energy harvesting. The design of an energy harvesting device must be tailored to the ambient energy available. For single frequency ambient excitation the resonant harvesting device is optimum, provided it is tuned to the excitation frequency. Several authors ${ }^{8,14-16}$ have proposed methods to optimize the parameters of the system to maximize the harvested energy. Shu et al. ${ }^{17-19}$ conducted detailed analysis of the power output for piezoelectric energy harvesting systems.

Regardless of the transduction mechanism, a primary issue in vibration-based energy harvesting is that the best performance of a generator is usually limited to excitation at its fundamental resonance frequency. If the applied ambient vibration deviates slightly from the resonance condition then the power output is drastically reduced. Hence a major issue in energy harvesting is to enable broadband energy harvesters. ${ }^{20,21}$ Thus researchers have recently focused on the concept of broadband energy harvesting to solve this issue with different approaches.

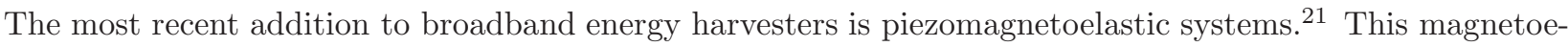
lastic structure (described in the following section) was first investigated by Moon and Holmes ${ }^{22}$ as a mechanical structure that exhibits strange attractor motions. Erturk et al. ${ }^{21}$ investigated the potential of this device for energy harvesting when the excitation is harmonic and demonstrated an order of magnitude larger power output over the linear system (without magnets) for non-resonant excitation. Litak et al. ${ }^{23}$ considered the performance of the nonlinear piezomagnetoelastic system to random excitations using numerical methods.

The theoretical analysis of piezomagnetoelastic systems is absent in the literature. The exact analysis of nonlinear piezomagnetoelastic energy harvesting system under random excitation requires the solution of the multidimensional Fokker-Planck equation to obtain the governing the probability density function of the harvested power. This paper considers a different approach and stochastically linearizes the system and develops an equivalent model of the overall system. The linear model is then analyzed to determine probability density functions of the system response and the power scavenged by the system. The paper is organized as follows: The next section describes the piezomagnetoelastic harvester. Details of the stochastic linearization technique and the three nonlinear equations that are required to be solved to obtain the equivalent linear system are reported in Section 3. The equivalent linear system under zero mean white noise excitation is analyzed in Section 4. Numerical results are reported in Section 5.

\section{MAGNETOPIEZOELASTIC ENERGY HARVESTERS}

Magnetopeizoelastic energy harvesters are the recent addition to energy harvesting devices. The device consists of a ferromagnetic cantilever beam that is excited at the support (see Figure 1). Two permanent magnets are located symmetrically on the base near the free end. The distance between the beam and the magnets determine the stable equilibrium points. In this article we considered interested in the case when the system has three equilibrium positions, two of which are stable, and the mechanical system is characterized by the classical double well potential. The nondimensional equations of motion for this system are

$$
\begin{gathered}
\ddot{x}+2 \zeta \dot{x}-\frac{1}{2} x\left(1-x^{2}\right)-\chi v=f(t), \\
\dot{v}+\lambda v+\kappa \dot{x}=0
\end{gathered}
$$

where $x$ is the dimensionless transverse displacement of the beam tip, $v$ is the dimensionless voltage across the load resistor, $\chi$ is the dimensionless piezoelectric coupling term in the mechanical equation, $\kappa$ is the dimensionless piezoelectric coupling term in the electrical equation, $\lambda \propto 1 / R_{l} C_{p}$ is the reciprocal of the dimensionless time constant of the electrical circuit, $R_{l}$ is the load resistance, and $C_{p}$ is the capacitance of the piezoelectric material. The force $f(t)$ is proportional to the base acceleration on the device. A simulation of the nonlinear system for random base excitation is shown in Figure 2. Gaussian white noise excitation, with zero mean and specified variance, was assumed for the simulations. Different values of the standard deviation of the excitation are considered.

Figure 2(a) shows the phase portrait for the excitation standard deviation $\sigma_{f}=0.03$. The system remains oscillating in one of its stable equilibrium point $x= \pm 1$ and never jumps to the other equilibrium point. This means that the excitation is too weak to provide sufficient energy to the system to overcome the potential barrier

Proc. of SPIE Vol. 797779770 N-2 


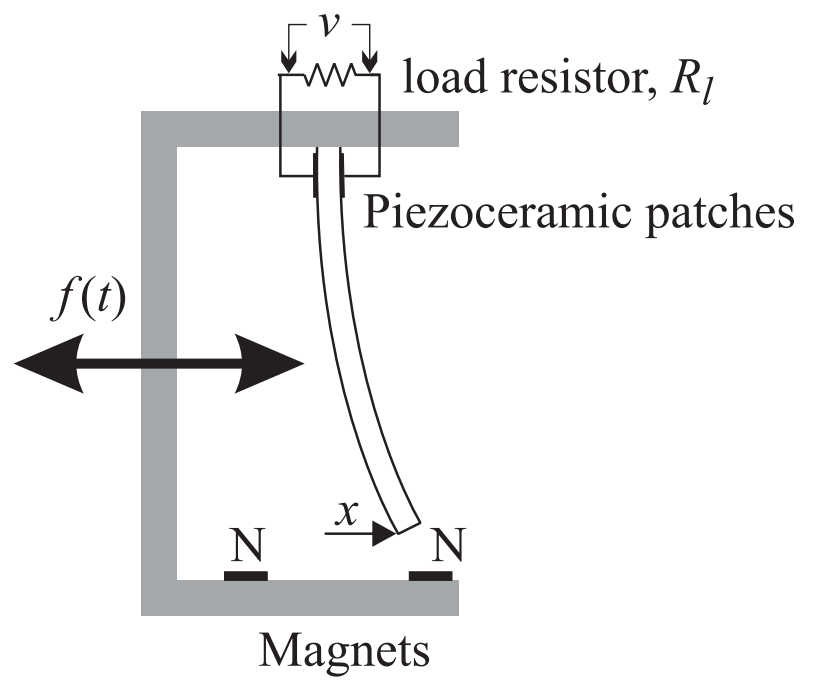

Figure 1. Schematic diagram of Magnetopiezoelastic device ${ }^{21}$

and jump to the other equilibrium point. When the standard deviation of the excitation is increases to $\sigma_{f}=0.07$ (i.e., Figure 2(b)) the system is seen to overcome this potential barrier and can jump to the other equilibrium point providing sufficient energy for scavenging. Next is when the standard deviation of excitation is increased to $\sigma_{f}=0.10$ the system seems to jump frequently between its equilibrium point providing more energy for scavenging.

Figure 2 demonstrates that the dynamics of the system is a double well potential and the greatest energy generation occurs when the system moves between the potential wells. To better understand this fact and get insight to the associated phenomenon this papers develops a equivalent linear system of the nonlinear system.

\section{STOCHASTIC EQUIVALENT LINEARIZATION}

Equation (1) is a nonlinear equation with nonlinearity in the stiffness term, whereas Equation (2) is a linear equation. To facilitate the linearization process, Equation (1) can be rewritten as

$$
\ddot{x}+2 \zeta \dot{x}+g(x)-\chi v=f(t)
$$

The nonlinear stiffness is represented as $g(x)=-\frac{1}{2}\left(x-x^{3}\right)$. Here we develop a linearized model for the Duffing equation (Equation (3)) based on the stochastic linearization approach. ${ }^{24}$ Assuming a non-zero mean random excitation (i.e., $f(t)=f_{0}(t)+m_{f}$ ) and a non-zero mean system response (i.e., $x(t)=x_{0}(t)+m_{x}$ ), the following equivalent linear system is considered,

$$
\ddot{x_{0}}+2 \zeta \dot{x_{0}}+a_{0} x_{0}+b_{0}-\chi v=f_{0}(t)+m_{f}
$$

where $f_{0}(t)$ and $x_{0}(t)$ are zero mean random processes. $m_{f}$ and $m_{x}$ are the mean of the original processes $f(t)$ and $x(t)$ respectively. $a_{0}$ and $b_{0}$ are the constants to be determined with $b_{0}=m_{f}$ and $a_{0}$ represents the square of the natural frequency of the linearized system $\omega_{e q}^{2}$. The idea is to substitute the nonlinear part, $g(x)$ with a linear stiffness parameter, $\left(\omega_{e q}^{2} x_{0}\right)$ in presence of random excitation $f_{0}(t)$.

Least squares optimization is used to minimize the expectation of the error norm i.e., $\left(\mathbb{E}\left[\epsilon^{2}\right]\right.$, with $\epsilon=$ $\left.g(x)-a_{0} x_{0}-b_{0}\right)$. The constants $a_{0}$ and $b_{0}$ are obtained in terms of the statistics of the response $x$. We take partial derivatives of the error norm w.r.t. $a_{0}$ and $b_{0}$ and equate them to zero individually. Finally we get,

$$
\begin{aligned}
& a_{0}=\frac{\mathrm{E}\left[g(x) x_{0}\right]}{\mathrm{E}\left[x_{0}^{2}\right]}=\frac{\mathrm{E}\left[g(x) x_{0}\right]}{\sigma_{x}^{2}} \\
& b_{0}=\mathrm{E}[g(x)]=m_{f}
\end{aligned}
$$




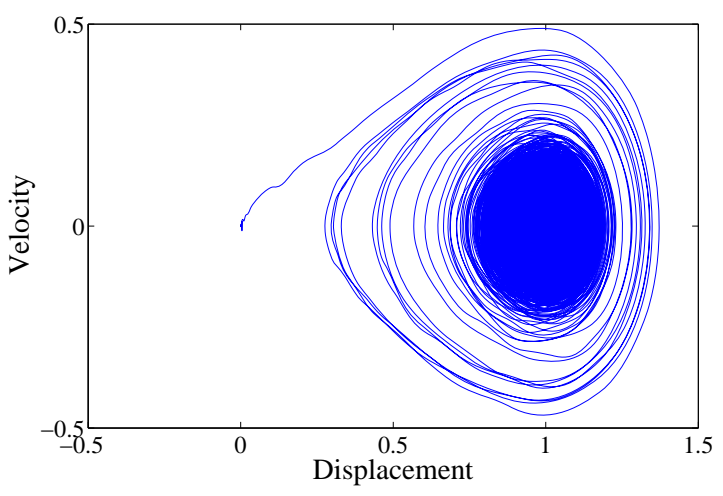

(a) Phase plane for $\sigma_{f}=0.03$

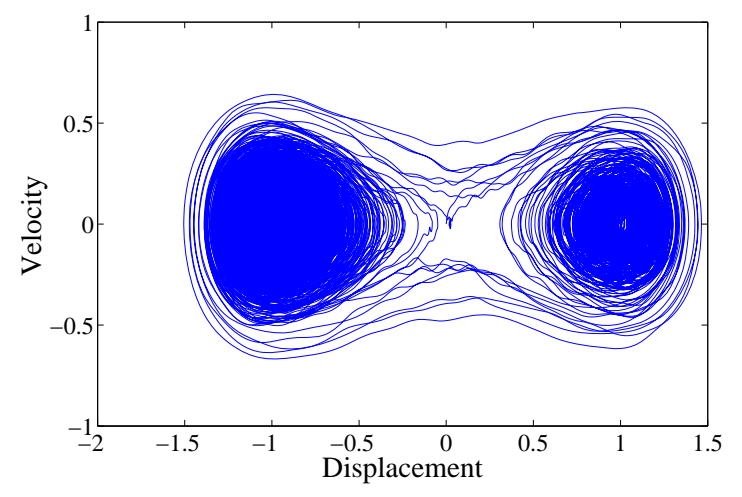

(b) Phase plane for $\sigma_{f}=0.07$

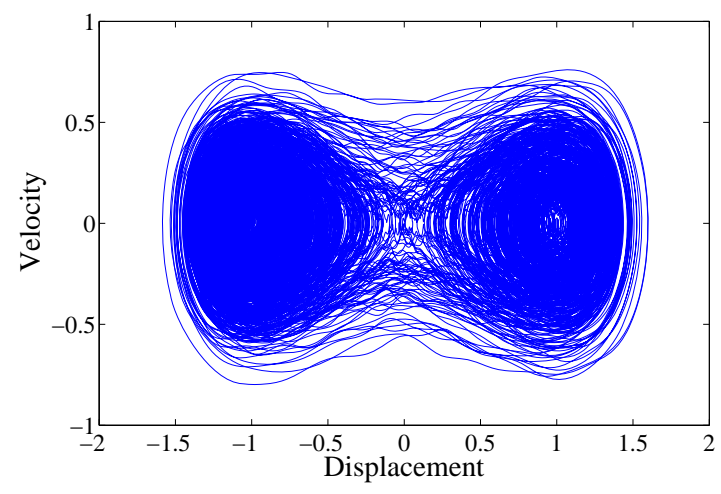

(c) Phase plane for $\sigma_{f}=0.10$

Figure 2. Phase plane diagram of the magnetopiezoelastic harvester for for $\lambda=0.01$ and for different standard deviation of the excitation a) $\sigma_{f}=0.03$, b) $\sigma_{f}=0.07$ and c) $\sigma_{f}=0.10$.

As a special case if we assume that $x(t)$ is a Gaussian random process, the expressions in Equations (5) and (6) can be further simplified ${ }^{24,25}$ as, (for details refer Ali et al. ${ }^{26}$ )

$$
a_{0}=-\frac{1}{2}\left\{1-3 \sigma_{x}^{2}-3 m_{x}^{2}\right\}
$$

which gives

$$
3 m_{x}^{2}+3 \sigma_{x}^{2}-2 a_{0}-1=0
$$

and

$$
m_{f}=-\frac{1}{2}\left(\mathrm{E}[x]-\mathrm{E}\left[x^{3}\right]\right) .
$$

which gives

$$
m_{x}\left(m_{x}^{2}+3 \sigma_{x}^{2}-1\right)-2 m_{f}=0
$$

where $m_{x}$ and $\sigma_{x}$ are the mean and standard deviation of the system response $x$.

The process of statistical linearization reduces to finding three unknowns $a_{0}, m_{x}$ and $\sigma_{x}$ with only two equations (Equations (8) and (10)). Another expression for $\sigma_{x}$ can be obtained from the linearized system equations. 
Equation (4) along with Equation (2) can be rewritten as,

$$
\begin{aligned}
\ddot{x_{0}}+2 \zeta \dot{x_{0}}+a_{0} x_{0}-\chi v & =f_{0}(t) \\
\dot{v}+\lambda v+\kappa \dot{x} & =0
\end{aligned}
$$

Taking the Fourier transform of Equations (11) and (12), we get,

$$
\left[\begin{array}{cc}
\left(a_{0}-\Omega^{2}\right)+2 \mathrm{i} \Omega \zeta & -\chi \\
\mathrm{i} \Omega \kappa & (\mathrm{i} \Omega+\lambda)
\end{array}\right]\left\{\begin{array}{l}
X(\Omega) \\
V(\Omega)
\end{array}\right\}=\left\{\begin{array}{c}
F_{0}(\Omega) \\
0
\end{array}\right\}
$$

Inverting the coefficient matrix, the displacement and voltage in the frequency domain can be obtained as

$$
\begin{aligned}
\left\{\begin{array}{l}
X \\
V
\end{array}\right\} & =\frac{1}{\Delta}\left[\begin{array}{cc}
(\mathrm{i} \Omega+\lambda) & \chi \\
-\mathrm{i} \Omega \kappa & \left(a_{0}-\Omega^{2}\right)+2 \mathrm{i} \Omega \zeta
\end{array}\right]\left\{\begin{array}{c}
F_{0} \\
0
\end{array}\right\} \\
& =H(\Omega)\left\{\begin{array}{c}
F_{0} \\
0
\end{array}\right\}
\end{aligned}
$$

where $H(\Omega)$ is the $2 \times 2$ matrix of frequency response functions and the determinant of the coefficient matrix is

$$
\Delta(\mathrm{i} \Omega)=(\mathrm{i} \Omega)^{3}+(2 \zeta+\lambda)(\mathrm{i} \Omega)^{2}+\left(2 \zeta \lambda+\kappa \chi+a_{0}\right)(\mathrm{i} \Omega)+\lambda a_{0}
$$

Since $f_{0}(t)$ is a weakly stationary, Gaussian, broadband random process, its autocorrelation function depends only on the difference in the time instants, and thus

$$
\mathrm{E}\left[f_{0}\left(\tau_{1}\right) f_{0}\left(\tau_{2}\right)\right]=R_{f_{0} f_{0}}\left(\tau_{1}-\tau_{2}\right)
$$

This autocorrelation function can be expressed as the inverse Fourier transform of the spectral density $\Phi_{f_{0} f_{0}}(\Omega)$ as

$$
R_{f_{0} f_{0}}\left(\tau_{1}-\tau_{2}\right)=\int_{-\infty}^{\infty} \Phi_{f_{0} f_{0}}(\Omega) \exp \left[\mathrm{i} \Omega\left(\tau_{1}-\tau_{2}\right)\right] \mathrm{d} \Omega .
$$

For a damped linear system of the form shown in Equation (15), it can be shown that ${ }^{24,27}$ the spectral density of $X(\Omega)$ is related to the spectral density of the excitation $F_{0}$ by

$$
\Phi_{x_{0} x_{0}}(\Omega)=\left|H_{11}(\Omega)\right|^{2} \Phi_{f_{0} f_{0}}(\Omega) .
$$

where $H_{11}(\Omega)$ is the element in the first row and column of the matrix $H(\Omega)$. In this paper we are interested in the standard deviation of response, $\sigma_{x}$, given as

$$
\sigma_{x}^{2}=\sigma_{x 0}^{2}=\int_{-\infty}^{\infty}\left|H_{11}(\Omega)\right|^{2} \Phi_{f_{0} f_{0}} \mathrm{~d} \Omega
$$

where $\Phi_{f_{0} f_{0}}$ is a constant for weakly stationary, white noise process.

Combining Equations (14), (15), (16) and (20), we get

$$
\sigma_{x}^{2}=\Phi_{f_{0} f_{0}} \int_{-\infty}^{\infty} \frac{\lambda^{2}+\Omega^{2}}{\Delta(\Omega) \Delta^{*}(\Omega)} \mathrm{d} \Omega
$$

Solving Equation (21) and then simplifying the resulting expression we obtain the final relation between $a_{0}$ and $\sigma_{x}$ (for details see Ali et al. ${ }^{26}$ ) and which is given as

$$
a_{0} \sigma_{x}^{2}\left(4 \lambda \zeta^{2}+2 \zeta \kappa \chi+2 \zeta a_{0}+2 \lambda^{2} \zeta+\lambda \kappa \chi\right)-\pi \Phi_{f_{0} f_{0}}\left(a_{0}+2 \lambda \zeta+\lambda^{2}\right)=0
$$

Equation (22) along with Equations (8) and (10) provide three equations to solve for the unknown variables $a_{0}$, $\sigma_{x}$, and $m_{x}$. Analytical solutions of Equations (8), (10) and (22) are not possible and one should make use of numerical schemes. In the next section, solutions for zero mean white noise excitation is shown. To summarise, one has to solve for $m_{x}, \sigma_{x}$ and $a_{0}$ from the following three nonlinear coupled equations,

$$
\begin{gathered}
3 m_{x}^{2}+3 \sigma_{x}^{2}-2 a_{0}-1=0 \\
m_{x}\left(m_{x}^{2}+3 \sigma_{x}^{2}-1\right)-2 m_{f}=0 \\
a_{0} \sigma_{x}^{2}\left(4 \lambda \zeta^{2}+2 \zeta \kappa \chi+2 \zeta a_{0}+2 \lambda^{2} \zeta+\lambda \kappa \chi\right)-\pi \Phi_{f_{0} f_{0}}\left(a_{0}+2 \lambda \zeta+\lambda^{2}\right)=0
\end{gathered}
$$




\section{ZERO MEAN WHITE NOISE EXCITATION}

\subsection{Determination of $\sigma_{x}, a_{0}$ and $m_{x}$}

Without loss of generality the external excitation can be assumed to be a zero mean white noise process, i.e., $m_{f}=0$. This largely simplifies our analysis and provides simple relation between the mean $\left(m_{x}\right)$ and the standard deviation $\left(\sigma_{x}\right)$ of the response. Putting $m_{f}=0$ in Equation (10) we get,

$$
m_{x}\left(m_{x}^{2}+3 \sigma_{x}^{2}-1\right)=0
$$

which gives, either $m_{x}=0$ or $m_{x}=\sqrt{1-3 \sigma_{x}^{2}}$.

Substituting $m_{x}=0$ in Equation (8) we get,

$$
3 \sigma_{x}^{2}=1+2 a_{0}
$$

Equation (25) shows that for $m_{x}=0$ and for any real $a_{0} \geq 0$, we have $\sigma_{x}^{2} \geq \frac{1}{3}$.

Substituting $m_{x}=\sqrt{1-3 \sigma_{x}^{2}}$ in Equation (8) we get,

$$
\begin{aligned}
3\left(1-3 \sigma_{x}^{2}\right)+3 \sigma_{x}^{2}-2 a_{0}-1 & =0 \\
1-3 \sigma_{x}^{2}-a_{0} & =0
\end{aligned}
$$

which gives

$$
3 \sigma_{x}^{2}=1-a_{0}
$$

Equation (27) shows that for $m_{x}^{2}=1-3 \sigma_{x}^{2}$ and real, positive $a_{0}$, we have $\sigma_{x}^{2} \in\left[0, \frac{1}{3}\right]$.

This on the other hand bounds $a_{0} \in[0,1]$. A further analysis will show that $a_{0}=m_{x}^{2}$.

Substituting Equation (25) and Equation (27) separately in Equation (22) we obtain the following two cubic polynomial equations respectively.

$$
\begin{aligned}
& 4 \zeta a_{0}{ }^{3}+\left(8 \lambda \zeta^{2}+4 \lambda^{2} \zeta+4 \kappa \chi \zeta+2 \zeta+2 \lambda \kappa \chi\right) a_{0}{ }^{2} \\
& \quad+\left(4 \lambda \zeta^{2}+2 \lambda^{2} \zeta+2 \kappa \chi \zeta+\lambda \kappa \chi-3 \Phi_{f_{0} f_{0}} \pi\right) a_{0}-3 \Phi_{f_{0} f_{0}} \pi \lambda(\lambda+2 \zeta)=0 \\
& 2 \zeta a_{0}{ }^{3}+\left(4 \lambda \zeta^{2}+2 \lambda^{2} \zeta+2 \kappa \chi \zeta-2 \zeta+\lambda \kappa \chi\right) a_{0}{ }^{2} \\
& \quad-\left(4 \lambda \zeta^{2}+2 \lambda^{2} \zeta+2 \kappa \chi \zeta+\lambda \kappa \chi-3 \Phi_{f_{0} f_{0}} \pi\right) a_{0}+3 \Phi_{f_{0} f_{0}} \pi \lambda(\lambda+2 \zeta)=0
\end{aligned}
$$

Numerical solutions show that Equation (28) has a single real positive root and Equation (29) has two real positive roots for any given value of $\Phi_{f_{0} f_{0}}$.

\subsection{Determination of $\mathrm{E}\left[v^{2}\right]$}

The spectral density of the voltage generated across the harvester can be related to the excitation as

$$
\Phi_{v v}(\Omega)=\left|H_{21}(\Omega)\right|^{2} \Phi_{f_{0} f_{0}}(\Omega) .
$$

where $H_{21}(\Omega)$ is the element in the second row and first column of the matrix $H(\Omega)$ (see Equation (14)).

$$
\mathrm{E}\left[v^{2}\right]=\int_{-\infty}^{\infty}\left|H_{21}(\Omega)\right|^{2} \Phi_{f_{0} f_{0}} \mathrm{~d} \Omega
$$

where $\Phi_{f_{0} f_{0}}$ is a constant for weakly stationary, white noise process.

Combining Equations (14), (15), (16) and (31), we get

$$
\mathrm{E}\left[v^{2}\right]=\Phi_{f_{0} f_{0}} \int_{-\infty}^{\infty} \frac{\kappa^{2} \Omega^{2}}{\Delta(\Omega) \Delta^{*}(\Omega)} \mathrm{d} \Omega
$$

Proceeding as shown in Ali et al., ${ }^{26}$ we find an expression for $\mathrm{E}\left[v^{2}\right]$ as

$$
\mathrm{E}\left[v^{2}\right]=\frac{\kappa^{2} \lambda a_{0}}{a_{0}\left(4 \lambda \zeta^{2}+2 \zeta \kappa \chi+2 \zeta a_{0}+2 \lambda^{2} \zeta+\lambda \kappa \chi\right)} \pi \Phi_{f_{0} f_{0}}
$$




\section{NUMERICAL ANALYSIS}

As discussed in Section 4.1, the analytical solution of Equations (28) and (29) is not feasible. Numerical methods are used to solve for different values of $\sigma_{x}, a_{0}$ and $m_{x}$. The solutions of Equations (28) and (29) for various values of standard deviation of the excitation are reported.

The system parameters have been taken as follows: ${ }^{21} \zeta=0.01, \chi=0.05$, and $\kappa=0.5$, while $\lambda$ was varied between 0.01 and 0.05 . The excitation $f(t)$ is considered stationary Gaussian white noise with standard deviation $\sigma_{f}$ ranging from $1 \%$ to $11 \%$. Higher values of standard deviation are not considered as statistical linearization would fail to represent the system for higher standard deviations.

The nonlinear piezomagnetoelastic system represents a Duffing type equation of motion. The nonlinear system has three equilibrium points, two stable equilibrium points at $x= \pm 1$ and one unstable equilibrium point at the origin $(x=0)$. Numerical simulations show that Equation $(28)$ has a single real positive solution for $a_{0}$, whereas Equation (29) has two real positive solutions. The solutions for different standard deviations of excitation are shown in Figure 3. 'Solution-1' and 'Solution-2' are the solutions for Equation (29) i.e., where $m_{x}=\sqrt{1-3 \sigma_{x}^{2}}$, and 'Solution-3' is obtained solving Equation (28) i.e., solution representing zero mean response. This representation of the solutions is true for all figures reported in this article.

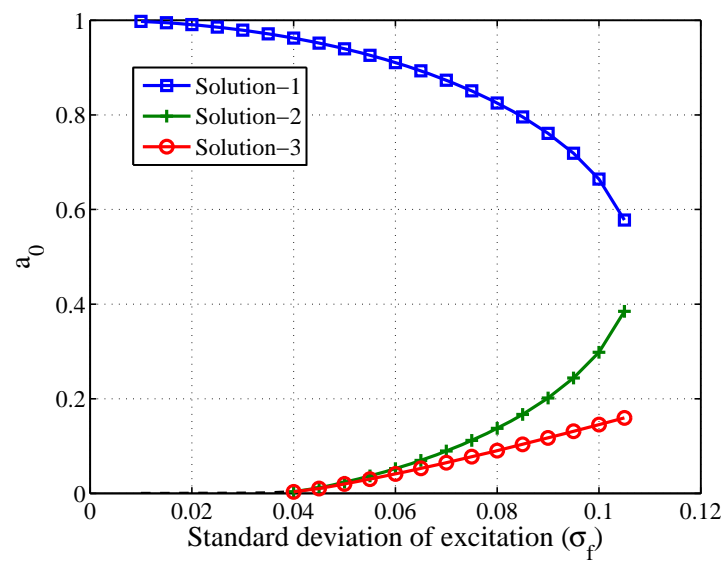

(a) $a_{0}$ for $\lambda=0.01$

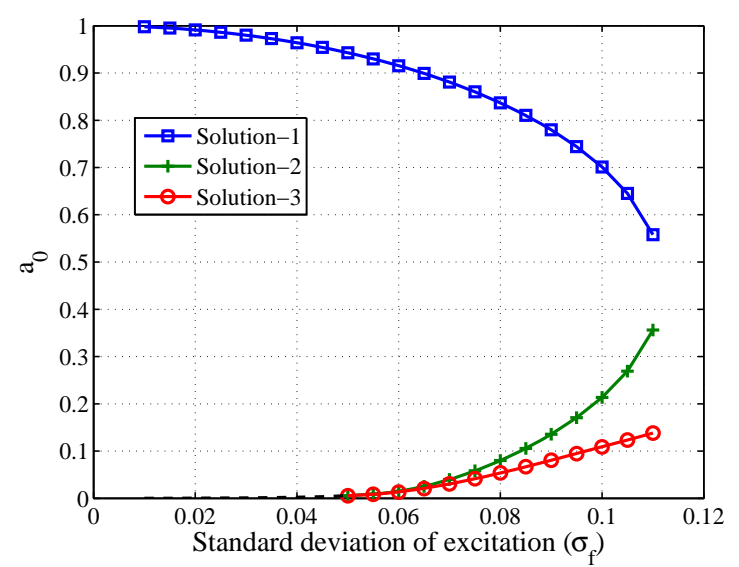

(b) $a_{0}$ for $\lambda=0.05$

Figure 3. Square of the natural frequency of the equivalent linear system for different standard deviations of the excitation $\left(\sigma_{f}\right)$ for a) $\lambda=0.01$ and b) $\lambda=0.05$.

Figures 3(a) and 3(b) are for $\lambda=0.01$ and $\lambda=0.05$ respectively. Note that $a_{0}$ is the square of the natural frequency of the equivalent linear system (see Equation (4)) and therefore can not be negative. The blue curve (with square marker) represents both the $x= \pm 1$ stable equilibrium points. The black dasched line represents the solution $a_{0}=0$ up to $\sigma_{f}=0.04$ and $\sigma_{f}=0.05$ for $\lambda=0.01$ and $\lambda=0.05$ respectively. This solution is the unstable equilibrium point of the system. Therefore the only practical solution for the system below $\sigma_{f}=0.04$ for electrical constant $\lambda=0.01$ and below $\sigma_{f}=0.05$ for $\lambda=0.05$ is given by the blue curve with square marker. At this stage the system remains in one of the potential wells represented by $x= \pm 1$.

When the excitation standard deviation reaches the 'cut-off' ( $\sigma_{f}=0.04$ for $\lambda=0.01$ and $\sigma_{f}=0.05$ for $\lambda=0.05)$ the system has enough energy to cross the potential barrier and could jump to the other potential well. This jump from one potential well to another generates more energy as will be shown later. These 'cut-off' standard deviations of the excitation are explained as 'the stochastic resonance phenomenon' in Litak et al. ${ }^{23}$

Figure 4 shows the square of the standard deviation of the response shown against the standard deviation of the excitation. It can be observed (and as described in the Section 4.1) that two real positive solutions of $\sigma_{x}^{2}$ exists for $m_{x} \neq 0$ which have values less than $\frac{1}{3}$. Another real positive solution is obtained solving Equation (28) which results into $\sigma_{x} \geq \frac{1}{3}$. 


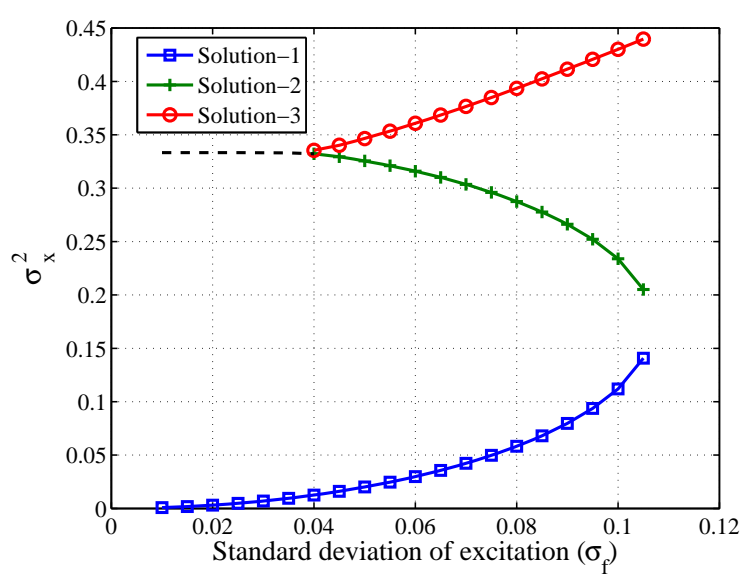

(a) Statistical response for $\lambda=0.01$

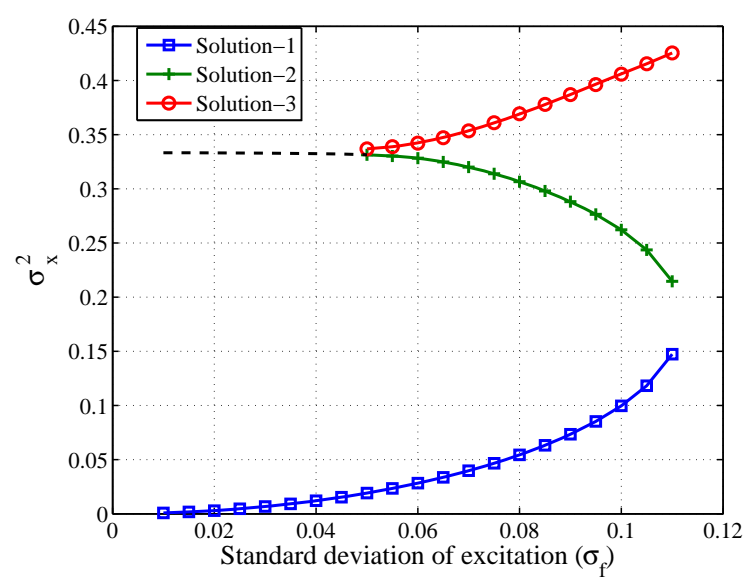

(b) Statistical response for $\lambda=0.05$

Figure 4. Square of standard deviation of the response under different standard deviations of the excitation for a) $\lambda=0.01$ and b) $\lambda=0.05$. The black dotted line represents unstable solution at $a_{0}=0$.

Note that the square of the standard deviation of the response $\sigma_{x}^{2}=\frac{1}{3}$ for 'Solution-2' and 'Solution-3' as long as the standard deviation of excitation $\sigma_{f} \leq 0.04$ and $\sigma_{f} \leq 0.05$ for $\lambda=0.04$ and $\lambda=0.05$ respectively. At the 'cut-off' standard deviation of excitation the standard deviation of response could jump from 'Solution-1' to 'Solution-2' or 'Solution-3' (depending on the initial condition of the system) generating more power. This phenomenon is observed in the solution of the nonlinear system as shown in Litak et al. ${ }^{23}$ Another point to note is that the $\sigma_{x}^{2}$ curves are similar for both $\lambda=0.04$ and $\lambda=0.05$, the only distinction being the 'cut-off' value of $\sigma_{f}$. This shows that electrical constant $(\lambda)$ plays little role in the response of the system and the mechanical and electrical systems are only weakly coupled.

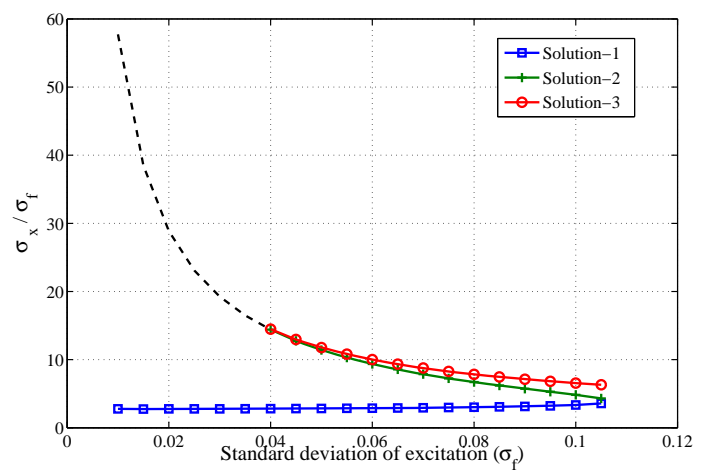

(a) Ratio of response to excitation for $\lambda=0.01$

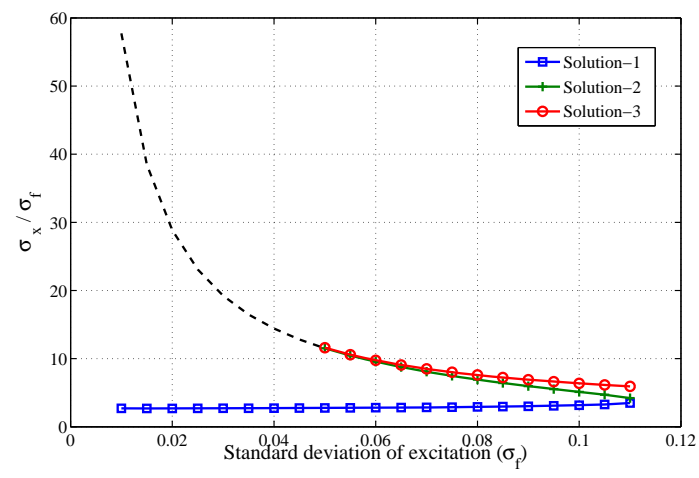

(b) Ratio of response to excitation for $\lambda=0.05$

Figure 5. Ratio of standard deviation of displacement to standard deviation of the excitation under different standard deviations of the excitation for a) $\lambda=0.01$ and b) $\lambda=0.05$. The black dotted line represents the unstable solution at $a_{0}=0$.

Figure 5 shows the ratio of standard deviations of the response and the excitation. An order increase in the standard deviation of the response is seen near the 'cut-off' region. As described earlier the third solution is the zero mean response. Interestingly the mean responses seem to be converging to a particular value with increase in standard deviation of excitation. As the excitation noise is increased the oscillation of the magnetoelastic rod crosses the potential barrier between individual equilibria and keeps oscillating. Therefore the equivalent 
linearization does not represent any equilibrium value at higher excitation deviations rather it shows a new point over which the system keeps on oscillating.

The variance of the voltage which is a measure of the mean power harvested is shown in Figure 6 for $\lambda=0.01$ and $\lambda=0.05$. Figures $6(\mathrm{a})$ and $6(\mathrm{~b})$ show that the voltage increases across the circuit near the 'cut-off' values of the excitation standard deviation. Note that the power remains very low for noise intensities below the 'cut-off' value. The electrical constant $\lambda$ significantly affects the voltage produced and hence the power generated. Note that this increase in power above the 'cut-off' point' is due to the changes in the electrical system, since the mechanical responses shown in Figures 3 to 5 are almost identical. The practical implementation of a increase in $\lambda$ requires a reduction in either electrical resistance or capacitance. This matches the reality i.e, power increases with a decrease in resistance across the circuit. These requirements should be included in trade-off studies required for the design of a real system.

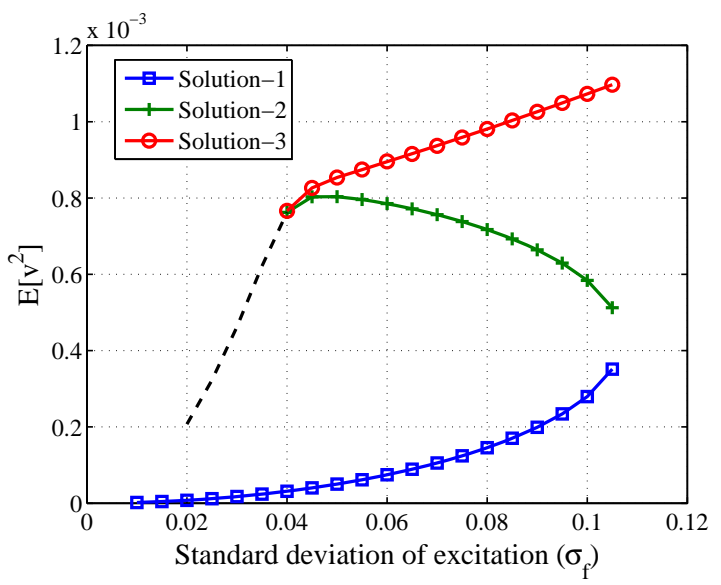

(a) Variance of voltage for $\lambda=0.01$

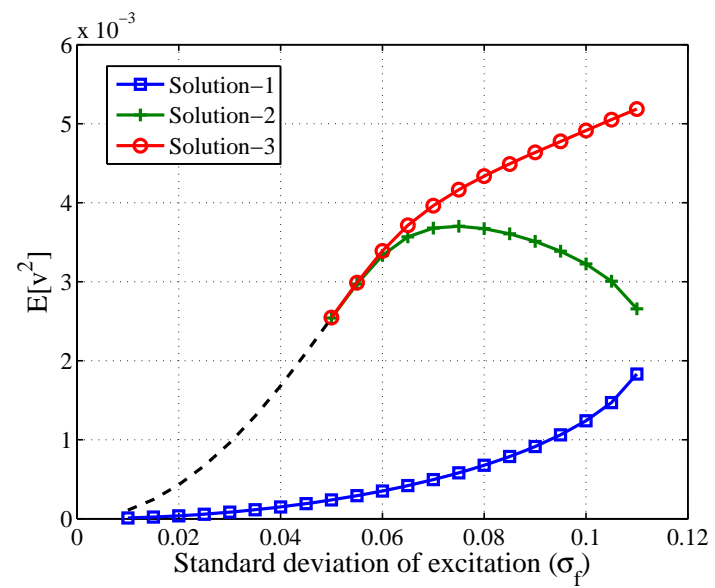

(b) Variance of voltage for $\lambda=0.05$

Figure 6 . Variance of voltage against the excitation standard deviation a) $\lambda=0.01$ and b) $\lambda=0.05$.

\section{CONCLUSION}

Piezomagnetoelectric harvester are best suited for broadband energy harvesting. These devices are nonlinear and their mechanical counterpart is represented by Duffing type oscillator. The device is nonlinear and can have chaotic motion depending on the input excitation. The chaotic motion generates an order of magnitude larger power output over the linear system (without magnets) for non-resonant excitation. This paper develops an equivalent linear model of the nonlinear system. An analytical expression for the equivalent linear system is given. It is observed that there exist a 'cut-off' standard deviation of the input excitation below which the power scavenged by the device is very low. The power generated by the device increases rapidly as the standard deviation of the input excitation is increased. This 'cut-off' standard deviation of the input excitation changes with the electrical constant of the system. The lower the electrical constant (i.e., higher $\lambda$ ), the higher is the 'cut-off' standard deviation. The effect of the variation in the electrical constant on the mechanical response of the system is seen to be relatively small. The power generating capacity of the system increases with increasing $\lambda$. Thus decreasing the resistance or capacitance of the system will increase the harvested power.

\section{Acknowledgement}

Dr. S. F. Ali gratefully acknowledges the support of Royal Society through Newton International Fellowship. 


\section{REFERENCES}

1. C. Williams and R. Yates, "Analysis of a micro-electric generator for microsystems," Sensors and Actuators A-Physical 52, pp. 8-11, 1996.

2. D. P. Arnold, "Review of microscale magnetic power generation," IEEE Transactions on Magnetics 43(11), pp. 3940-3951, 2007.

3. S. P. Beeby, R. N. Torah, M. J. Tudor, P. Glynne-Jones, T. O’Donnell, C. R. Saha, and S. Roy, "A micro electromagnetic generator for vibration energy harvesting," Journal of Micromechanics and Microengineering 17(7), pp. 1257-1265, 2007.

4. R. Amirtharajah and A. Chandrakasan, "Self-powered signal processing using vibration-based power generation," IEEE Journal of Solids State Circuits 33(5), pp. 687-695, 1998.

5. S. P. Beeby, M. J. Tudor, and N. M. White, "Energy harvesting vibration sources for microsystems applications," Measurement Science and Technology 17(12), pp. 175-195, 2006.

6. E. Halvorsen, "Energy harvesters driven by broadband random vibrations," J. of Microelectromechanical Systems 17(5), pp. 1061-01071, 2008.

7. H. A. Sodano, D. J. Inman, and G. Park, "A review of power harvesting from vibration using piezoelectric materials," The Shock and Vibration Digest 36(3), pp. 197-205, 2004.

8. N. E. Dutoit, B. L. Wardle, and S.-G. Kim, "Design consideration for mems scale piezoelectric mechanical vibration energy harvesters," Integrated Ferroelectrics An International Journal 71(1), pp. 121-160, 2005.

9. S. Priya, "Advances in energy harvesting using low profile piezoelectric transducers," J. of Electroceramics 19(1), pp. 167-184, 2007.

10. S. Ali, S. Adhikari, and M. I. Friswell, "Piezoelectric energy harvesting with parametric uncertainty," Smart Materials \&5 Structures 19(10), p. 105010, 2010.

11. H. A. Sodano, G. Park, and D. J. Inman, "Estimation of electric charge output for piezoelectric energy harvesting," Strain 40(2), pp. 49-58, 2004.

12. S. R. Anton and H. A. Sodano, "A review of power harvesting using piezoelectric materials (2003-2006)," Smart Materials and Structures 16(3), pp. R1-R21, 2007.

13. E. Lefeuvre, A. Badel, C. Richard, L. Petit, and D. Guyomar, "A comparison between several vibrationpowered piezoelectric generators for standalone systems," Sensors and Actuators A: Physical 126(2), pp. 405-416, 2006.

14. T. Ng and W. Liao, "Sensitivity analysis and energy harvesting for a self-powered piezoelectric sensor," Journal of Intelligent Material Systems and Structures 16(10), pp. 785-797, 2005.

15. S. Roundy, "On the effectiveness of vibration-based energy harvesting," Journal of Intelligent Material Systems and Structures 16(10), pp. 809-823, 2005.

16. J. M. Renno, M. F. Daqaq, and D. J. Inman, "On the optimal energy harvesting from a vibration source," Journal of Sound and Vibration 320(1-2), pp. 386-405, 2009.

17. Y. Shu and I. Lien, "Analysis of power output for piezoelectric energy harvesting systems," Smart Materials \& Structures 15(6), pp. 1499-1512, 2006.

18. Y. Shu and I. Lien, "Efficiency of energy conversion for a piezoelectric power harvesting system," J. of Micromechanics and Microengineering 16(11), pp. 2429-2438, 2006.

19. Y. C. Shu, I. Lien, and W. Wu, "An improved analysis of the sshi interface in piezoelectric energy harvesting," Smart Materials $\mathcal{E} 3$ Structures 16(6), pp. 2253-2264, 2007.

20. B. Marinkovic and H. Koser, "Smart sand-a wide bandwidth vibration energy harvesting platform," Applied Physics Letters 94(10), p. 103505, 2009.

21. A. Erturk, J. Hoffmann, and D. J. Inman, "A piezomagnetoelastic structure for broadband vibration energy harvesting," Applied Physics Letters 94((25)), p. 254102, 2009.

22. F. C. Moon and P. J. Holmes, "A magnetoelastic strange attractor," J. of Sound and Vibration 65(2), pp. 275-296, 1979.

23. G. Litak, M. I. Friswell, and S. Adhikari, "Magnetopiezoelastic energy harvesting driven by random excitations," Applied Physics Letterss 96(21), p. 214103, 2010.

24. J. B. Roberts and P. D. Spanos, Random Vibration and Statistical Linearization, Dover Publications, Mineola, New York., 2003. 
25. I. E. Kazakov, "Generalization of methods of statistical linearizarion to multidimensional systems," $A u$ tomation and Remote Control 26, pp. 1201-1206, 1965.

26. S. Ali, S. Adhikari, M. I. Friswell, and S. Narayan, "The analysis of piezomagnetoelastic energy harvesters under broadband random excitations," Journal of Applied Physics (Accepted), 2011.

27. N. C. Nigam, Introduction to Random Vibration, The MIT Press, London, England, 1983.

28. S. Adhikari, M. I. Friswell, and D. J. Inman, "Piezoelectric energy harvesting from broadband random vibrations," Smart Materials and Structures 18(11), p. 115005, 2009. 